Check for updates

Cite this: RSC Adv., 2019, 9, 29474

Received 24th July 2019

Accepted 4th September 2019

DOI: 10.1039/c9ra05716a

rsc.li/rsc-advances

\section{Ultrathin nickel-metal-organic framework nanobelt based electrochemical sensor for the determination of urea in human body fluids $\dagger$}

\author{
Cancan Bao, ${ }^{a}$ Qiangqiang Niu, ${ }^{a}$ Zi-Ang Chen, ${ }^{a}$ Xiaowei Cao, ${ }^{1}{ }^{b}$ Hui Wang ${ }^{a}{ }^{a}$ \\ and Wenbo Lu (iD)*a
}

\begin{abstract}
Ultrathin nickel-metal-organic framework (Ni-MOF) nanobelts, $\left[\mathrm{Ni}_{20}\left(\mathrm{C}_{5} \mathrm{H}_{6} \mathrm{O}_{4}\right)_{20}\left(\mathrm{H}_{2} \mathrm{O}\right)_{8}\right] \cdot 40 \mathrm{H}_{2} \mathrm{O}(\mathrm{Ni}-\mathrm{MIL}-$ 77), have been exploited successfully for the fabrication of a non-enzymatic urea sensor. Ni-MOF ultrathin nanobelts in alkaline media can be used as an efficient catalyst for urea electrooxidation. As a non-enzymatic urea sensor, Ni-MOF ultrathin nanobelts exhibit a high sensitivity of $118.77 \mu \mathrm{A} \mathrm{mM} \mathrm{m}^{-1}$ $\mathrm{cm}^{-2}$, wide linear range of $0.01-7.0 \mathrm{mM}$, and low detection limit of $2.23 \mu \mathrm{M}(\mathrm{S} / \mathrm{N}=3)$. The selectivity, stability and reliability of ultrathin Ni-MOF nanobelts towards urea oxidation are also investigated. Moreover, Ni-MOF ultrathin nanobelts were further used to detect urea in human body fluids. All these findings confirm that the urea sensor based on Ni-MOF ultrathin nanobelts is successfully prepared and promising for applications in medical diagnostics and environmental monitoring.
\end{abstract}

\section{Introduction}

Urea is one of the primary products of protein degradation and metabolism of nitrogen containing compounds. ${ }^{1}$ The amount of urea present in human body fluids (such as blood and urine) exceeds a certain level, which can cause damage to kidney or liver of mankind. ${ }^{2}$ If the kidneys or livers are not working properly, the concentration of urea will be abnormal in the body fluids. $^{3}$ Abnormal levels of urea in human body fluids cause kidney or hepatic failure, nephritic syndrome and urinary tract obstruction. ${ }^{4}$ Furthermore, urea in industrial waste water is washed out in the environment, which can pollute the surface and the ground water., ${ }^{5,6}$ Accordingly, it is essential to monitor urea levels in the human body fluids, environment, and drinking water.

Metal-organic frameworks (MOFs) have some advantages, such as high surface area and tuneable chemical structures. ${ }^{7}$ Nickel-based nanomaterials have been extensively applied for the direct electrocatalytic oxidation of biochemical molecules. ${ }^{\mathbf{8}, 9}$ The electrocatalytic performance of the nickel-based catalysts has been further improved by preparing various nanostructures such as nanoparticles, ${ }^{10}$ nanorods,${ }^{11}$ nanowires,${ }^{12}$

${ }^{a}$ Key Laboratory of Magnetic Molecules and Magnetic Information Materials (Ministry of Education), School of Chemistry and Material Science, Shanxi Normal University, Linfen 041004, China. E-mail: luwb@sxnu.edu.cn; Fax: +86-357-2051192; Tel: +86357-2051192

${ }^{b}$ Institute of Translational Medicine, Medical College, Yangzhou University, Yangzhou 225001, China

$\dagger$ Electronic supplementary information (ESI) available. See DOI: 10.1039/c9ra05716a and three-dimensional networks. ${ }^{13,14}$ Nickel-based metalorganic frameworks (Ni-MOFs) have generated a large amount of interest because of their excellent structural and electrocatalytic properties. ${ }^{13}$ In particular, Ni-MOF based catalysts have exhibited excellent catalytic abilities for urea oxidation. ${ }^{15,16}$ However, few reports are available on the detection of urea by Ni-MOFs. Recently, Yoon's group synthesized Ni-MOF microparticle and multiwalled carbon nanotube composites for urea detection. ${ }^{17}$ Ni-MOF microparticles with a diameter of $1.2 \mu \mathrm{m}$ are too big to avoid many shortcomings. ${ }^{17}$ As a result, it is very necessary to develop a new class of Ni-MOF nanomaterials for the detection of urea. Ni-MOF nanobelts possess a rectangular area on each ribbon strip, which exhibit high electrocatalytic activity because of large surface-to-volume ratio and highly active surface. ${ }^{17}$ As far as we know, Ni-MOF nanobelts for the determination of urea have not yet been reported.

For the first time, we describe our finding that Ni-MOF ultrathin nanobelts, $\left[\mathrm{Ni}_{20}\left(\mathrm{C}_{5} \mathrm{H}_{6} \mathrm{O}_{4}\right)_{20}\left(\mathrm{H}_{2} \mathrm{O}\right)_{8}\right] \cdot 40 \mathrm{H}_{2} \mathrm{O}$ (Ni-MIL-77), in alkaline media can be used as an efficient catalyst for urea electrooxidation, as shown in this article. As a non-enzymatic urea sensor, Ni-MOF ultrathin nanobelts exhibit superior urea sensing performances, such as broad detection range, low detection limit and high selectivity. Moreover, the detection of urea in human body fluids has been further explored for the sensing performance of Ni-MOFs ultrathin nanobelts.

\section{Results and discussion}

\section{Physical characterization}

The morphology and structure of Ni-MOF ultrathin nanobelts (the Ni-MOF nanobelts are synthesised by a hydrothermal 
method using nickel acetate and glutaric acid as main raw materials, and the relevant experimental procedure is shown in the ESI $\dagger$ ) have been explored by scanning electron microscopy (SEM), transmission electron microscopy (TEM) and other characterization methods. The phase purity and crystallographic structure of the experimental product was characterized by XRD. As shown in Fig. S1 $\uparrow$ (before and after electrochemical testing), the peak intensity is stronger before electrochemical testing. However, the crystal plane of XRD did not change after electrochemical testing. Therefore, we can draw the conclusion that the two compounds before and after testing are the same substance, and the structure of Ni-MOF nanobelts is stable.

Fig. 1A shows the SEM images of Ni-MOF and reveals the formation of ultrathin nanobelts. The nanobelt structure can be further observed clearly in the inset of Fig. 1A. In addition, the SEM of Ni-MOF is also characterized after a long-term detection of $1 \mathrm{mM}$ urea in $0.1 \mathrm{M} \mathrm{KOH}$ as displayed in Fig. S2. $\dagger$ It can be seen that the morphology of Ni-MOF nanobelts has not changed substantially after long time detection, and its original nanobelt morphology is basically maintained. It again confirms the stability of Ni-MOF nanobelts. Fig. 1B exhibits the TEM images of Ni-MOFs from which we can achieve a deeper understanding of the structure of ultrathin nanobelts. The energy-dispersive Xray (EDX) spectrum exhibits the rational elemental presence of $\mathrm{Ni}, \mathrm{C}$ and $\mathrm{O}$ existing in the composites, which demonstrates that no impurity substance exists in the Ni-MOF nanobelts (Fig. 1C). The Fourier transform infrared (FT-IR) spectrum of the NiMOFs is revealed in Fig. 1D. The intensive and broad peak located at $3425 \mathrm{~cm}^{-1}$ is attributable to the stretching mode of the hydroxyl group (O-H) coupled to $\mathrm{Ni}(\mathrm{II})$, and the peaks located at $1309 \mathrm{~cm}^{-1}, 1453 \mathrm{~cm}^{-1}$ and $1413 \mathrm{~cm}^{-1}$ represent the $\mathrm{C}-\mathrm{H}$ bond vibrations. Moreover, an intensive band lies at $1606 \mathrm{~cm}^{-1}$ because of the representative pattern of alkene $(\mathrm{C}=$ C). Above results are consistent with the literature. ${ }^{18}$ The formation of Ni-MOFs is confirmed mediately indirectly by FTIR spectroscopy. We can identify the fundamental form and composition of functional groups of Ni-MOFs through X-ray photoelectron spectroscopy (XPS). As shown in Fig. 1E, the survey of XPS shows distinct peaks located at $856.6 \mathrm{eV}\left(\mathrm{Ni} 2 \mathrm{p}^{3}\right)$, $530.64 \mathrm{eV}(\mathrm{O} 1 \mathrm{~s})$ and $283.1 \mathrm{eV}$ (C 1s), separately, demonstrating
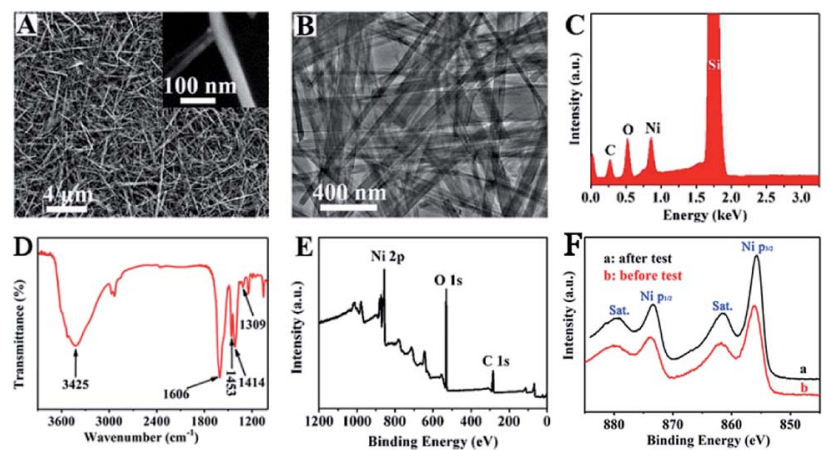

Fig. 1 Low (A) and high (the inset) magnification SEM images of $\mathrm{Ni}$ MOFs; (B) TEM image of Ni-MOFs; (C) EDX spectrum of Ni-MOFs; (D) FT-IR spectrum of Ni-MOFs; XPS patterns of survey (E) and Ni $2 p$ regions before and after testing (F) on the Ni-MOFs. that Ni-MOFs have been successfully synthesized. A series of visible peaks of Ni $2 \mathrm{p}$ region spectra can be viewed in Fig. 1F. Two peaks at $c a .856 .32$ and $873.65 \mathrm{eV}$ are assigned to $\mathrm{Ni} 2 \mathrm{p}_{1 / 2}$ and $\mathrm{Ni} 2 \mathrm{p}_{3 / 2}$, respectively. ${ }^{19,20}$ Moreover, the broad peaks at $861.69 \mathrm{eV}$ and $879.9 \mathrm{eV}$ represent the satellite peaks of Ni $2 \mathrm{p}_{1 / 2}$ and Ni $2 \mathrm{p}_{3 / 2}$, respectively. ${ }^{21}$ The results received from the Ni $2 \mathrm{p}$ spectra testified the fact that nickel ions consisted in the prepared Ni-MOFs are present in the form of Ni(II). When compared before and after urea electrooxidation in alkaline medium, it can be seen that the valence and peak position of $\mathrm{Ni}$ $2 p$ regions on Ni-MOF nanobelts has not changed substantially after a long-term detection. It again demonstrates that the structure of Ni-MOF nanobelts is stable. In conclusion, all of the above characterizations confirm that the Ni-MOF nanobelts have been prepared successfully.

\section{Electrochemical behaviors towards urea}

To estimate the electrocatalytic property of the Ni-MOF ultrathin nanobelts toward urea, cyclic voltammetric (CV) tests of GCE, Nafion/GCE and Ni-MOF/Nafion/GCE are carried out with and without $1.0 \mathrm{mM}$ urea (scan speed: $50 \mathrm{mV} \mathrm{s}^{-1}$ ). All electrooxidation behaviors of urea on the Ni-MOF/Nafion/GCE electrode in this experiment are studied in $0.1 \mathrm{M} \mathrm{KOH}$ electrolyte. As displayed in Fig. 2, a couple of characteristic redox peaks turned up immediately when the GCE surface was modified with Ni-MOF nanobelts (curve a and b); in comparison, bare GCE and Nafion/GCE show no typical peak with $1.0 \mathrm{mM}$ urea (curve $\mathrm{c}$ and d). From the CV diagram, we can see that there is no typical redox peak (curve d), indicating that bare GCE has no catalytic performance towards urea. After modifying Nafion to the surface of GCE, there is almost no change in current, manifesting that Nafion acts only as a mediator for the transport of electrons and immobilization..$^{22,23}$ On the other hand, it can be also discovered that there is a significant oxidation peak corresponding to the anode at about $0.60 \mathrm{~V}$, which is attributed

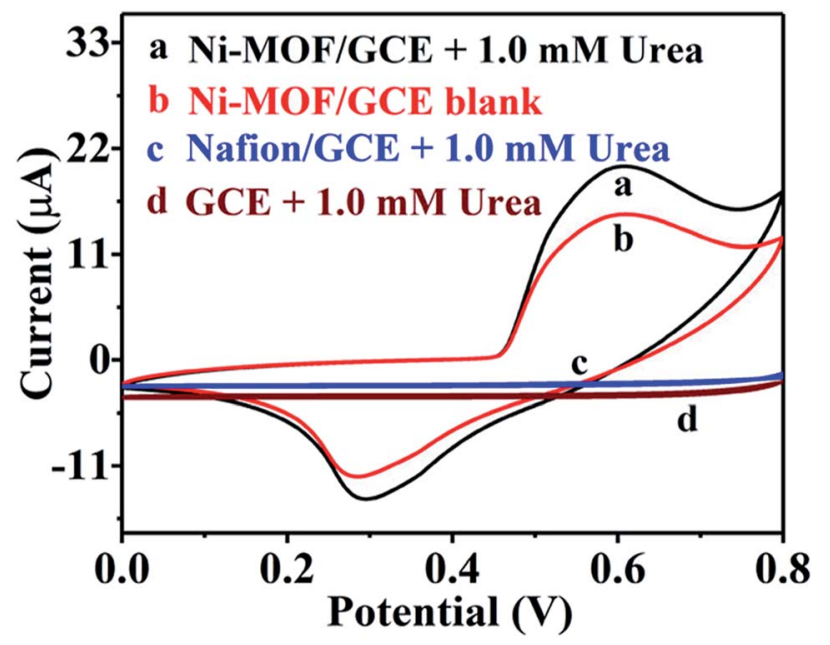

Fig. 2 Cyclic voltammograms of Ni-MOF/Nafion/GCE (curves a and b), Nafion/GCE (curve c) and GCE (curve d) electrodes with (curves a, C and d) and without (curve b) $1.0 \mathrm{mM}$ urea in $0.1 \mathrm{M} \mathrm{KOH}$ solution. Scan speed: $50 \mathrm{mV} \mathrm{s}^{-1}$. 
to the reason that $\mathrm{Ni(II)}$ can be rapidly converted to $\mathrm{Ni(III)} \mathrm{in}$ presence of large number of $\mathrm{OH}^{-}$anions. And the result is accordant with the literature. ${ }^{17}$ At the cathode, however, a visible reduction peak appears at about $0.30 \mathrm{~V}$, which can be considered as a process in which $\mathrm{Ni}(\mathrm{III})$ is reduced to $\mathrm{Ni}$ (II), where the process occurs opposite to the anode. At the same time, the peak current response heightens conspicuously in the anode and weakens in the cathode when $1.0 \mathrm{mM}$ urea was injected (curve a). The above detecting results demonstrate that Ni-MOF nanobelts possess an excellent electro-catalytic oxidation property towards urea. The above electrochemical behaviours are in accordance with the characteristic catalytic regeneration (EC' mechanism). ${ }^{24}$ Based on theory, urea is chemically oxidized to corresponding substances accompanied with the process of $\mathrm{Ni}$ (III) reduction to $\mathrm{Ni}(\mathrm{II})$, and the electrochemical regeneration of $\mathrm{Ni}$ (II) to Ni(III) occurs simultaneously. In other words, it's the following redox transition process: $\mathrm{Ni}(\mathrm{II})$ $\rightleftarrows \mathrm{Ni}(\mathrm{III})$. The regeneration of $\mathrm{Ni}(\mathrm{III})$ catalytic agents leads to the disappearance of invertibility and heightens the peak intensity of $\mathrm{Ni}($ III)/Ni(II) with the addition of urea.

It has been reported that a cost-effective nickel-based catalyst can convert urea into non-poisonous and harmless nitrogen gas products under alkaline conditions by electro-oxidation, ${ }^{25}$ and the electrocatalytic process of this material towards urea may occur as follows: $:^{8,17,20,26}$

$$
\begin{gathered}
\mathrm{Ni}(\mathrm{II})-\mathrm{MOF} \rightarrow \mathrm{Ni}(\mathrm{III})-\mathrm{MOF}+\mathrm{e}^{-} \\
\mathrm{Ni}(\mathrm{III})-\mathrm{MOF}+\mathrm{CO}\left(\mathrm{NH}_{2}\right)_{2}+6 \mathrm{OH}^{-} \rightarrow \\
\mathrm{Ni}(\mathrm{II})-\mathrm{MOF}+\mathrm{N}_{2}+5 \mathrm{H}_{2} \mathrm{O}+\mathrm{CO}_{2}+6 \mathrm{e}^{-}
\end{gathered}
$$

The above equations manifest that Ni(II) could be rapidly oxidized into active $\mathrm{Ni}$ (III) in an alkaline environment, which promotes the electrocatalytic oxidation procedure of urea. ${ }^{21}$

The structure of Ni-MOF is $\left[\mathrm{Ni}_{20}\left(\mathrm{C}_{5} \mathrm{H}_{6} \mathrm{O}_{4}\right)_{20}\left(\mathrm{H}_{2} \mathrm{O}\right)_{8}\right] \cdot 40 \mathrm{H}_{2} \mathrm{O}$. It can be seen that the ligand $\mathrm{C}_{5} \mathrm{H}_{6} \mathrm{O}_{4}$ is connected to Ni successively, and finally forms a series of nanobelt tunnels. When urea is added, it is absorbed on the Ni-MOF nanobelts. Under a certain voltage, urea undergoes an electrooxidation process in the Ni-MOF nanobelt tunnel. In the tunnel, Ni(II) could be rapidly oxidized into active $\mathrm{Ni}(\mathrm{III})$ under alkaline conditions, and the urea was oxidized into $\mathrm{N}_{2}, \mathrm{CO}_{2}$ and $\mathrm{H}_{2} \mathrm{O}$.

Furthermore, we studied the kinetic parameters of the process through a series of electroanalytical measurement techniques, including cyclic voltammetry (CV), linear sweep voltammetry (LSV), differential pulse voltammetry (DPV) and amperometric response curve $(I-T)$.

\section{Evaluation of electrochemical performances}

Effect of scanning speed. For an electrocatalytic reaction, it is exceedingly practicable to realize how the electrons are transferred in the course of the redox reaction, which helps us to have a deeper comprehension of the reaction process. Therefore, we investigated the influence of scanning speed on the peak current response of $\mathrm{Ni}(\mathrm{II}) / \mathrm{Ni}$ (III) redox couples by cyclic voltammetry (CV) and the result is shown in Fig. 3A. The response currents to the anode and cathode strengthened regularly in the wake of the
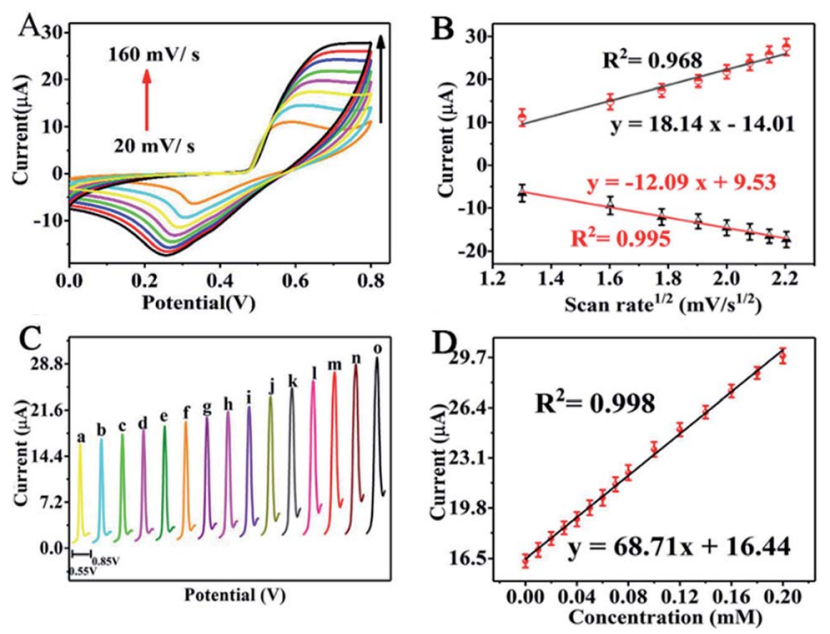

Fig. 3 (A) CVs of the Ni-MOF/Nafion/GCE electrode with $1.0 \mathrm{mM}$ urea at distinct scan speeds: $20,40,60,80,100,120,140,160 \mathrm{mV} \mathrm{s}^{-1}$. (B) The plot of variation of anode and cathode peak currents with scanning rate. (C) DPV of the Ni-MOF/Nafion/GCE electrode at variant urea concentrations: 0-0.2 mM. Test factors: amplitude $0.05 \mathrm{~V}$, pulse cycle time $0.2 \mathrm{~s}$ and impulse duration $0.05 \mathrm{~s}$. (D) The correlation of associated response $v s$. the concentration.

continually raised scanning speed. In addition, the redox peak current signals are linearly dependent on the the square root of the scan rates, as displayed in Fig. 3B. There is not only an excellent mutual dependence between the peak current of anodic oxidation and the radical sweep velocity, for which $R^{2}$ is 0.968 , but also an analogous relevance for the peak response to cathodic reduction, for which $R^{2}=0.995$. And the proportional relations existing between peak current and sweep velocity demonstrates that the redox reaction of urea on the Ni-MOF modified electrode is a representative diffusion - controlled process. ${ }^{17}$ In addition, the oxidation peak moves towards more positive direction corresponding to a series of increases in the scanning speed in the range of 20 to $160 \mathrm{mV} \mathrm{s}^{-1}$, while the reduction peak is completely opposite.

Moreover, the distance $\left(\Delta E_{\mathrm{p}}\right)$ between the peak potential of anode $\left(E_{\mathrm{pa}}\right)$ and cathode $\left(E_{\mathrm{pc}}\right)$ moves further away from each other ranging from about $248 \mathrm{mV}$ to $449 \mathrm{mV}$. The reason why the oxidation potential moves in the positive direction may be due to the extraordinary sorption to urea at the position of $\mathrm{Ni}\left(\right.$ III). ${ }^{27}$ Meanwhile, we can also observe that the peak signal response ratios $\left(I_{\mathrm{pc}} / I_{\mathrm{pa}}\right)$ between the cathode and the anode are explicitly departed from 1 , and the peak potential discrepancy $\left(\Delta E_{\mathrm{p}}\right)$ is larger than $0.059 / n$, manifesting the quasi-reversibility of the redox reaction.

On the other hand, there is a logarithmic relationship between the oxidation peak potential $\left(E_{\mathrm{pa}}\right)$ and sweep rate. The linear relationship (Fig. S3†) between $E_{\text {pa }}$ and $\log v$ can be demonstrated as follows:

$$
E_{\mathrm{pa}}(\mathrm{V})=0.794+0.136 \log v\left(\mathrm{~V} \mathrm{~s}^{-1}\right),\left(R^{2}=0.983\right)
$$

The electron transfer coefficient $(\alpha)$ can be obtained from the inferior formula between $E_{\mathrm{pa}}$ and $\log v:^{17}$ 


$$
E_{\mathrm{pa}}=\text { constant }+\frac{0.03}{\alpha n} \log v
$$

According to the slope of Fig. $\mathrm{S} 3, \uparrow \alpha$ can be figured out as 0.45 . The transferred electrons $(n)$ are calculated to be close to 6 . This indicates that 6 electrons are transferred on the anode during the electrochemical oxidation of urea. The total reaction is as follows:

$$
\mathrm{CO}\left(\mathrm{NH}_{2}\right)_{2}+6 \mathrm{OH}^{-} \rightarrow \mathrm{N}_{2}+5 \mathrm{H}_{2} \mathrm{O}+\mathrm{CO}_{2}+6 \mathrm{e}^{-}
$$

which is consistent with the mechanism of reactions described in the eqn (1) and (2).

Differential pulse voltammetry response of urea. The technique of differential pulse voltammetry (DPV) not only has higher sensitivity and resolution, but also has lower detection limit on account of the depressed background current, which can be employed in the situation where the concentration is as low as about $1.0 \mu \mathrm{g} \mathrm{L}^{-1}$. Fig. 3C reveals DPV response curves of the Ni-MOF decorated electrode in $0.1 \mathrm{M}$ basic electrolyte involving different urea concentrations: 0-2.0 $\mathrm{mM}$. Urea concentration was first increased stepwise by $0.1 \mathrm{mM}$ until it reached $0.8 \mathrm{mM}$, followed by $0.2 \mathrm{mM}$ in each step until it finally reached $2.0 \mathrm{mM}$. As presented in Fig. S4, $\dagger$ a clearer DPV curve can be examined when urea is not present in the potential range of 0.55-0.85 V vs. Ag/AgCl. Fig. 3D exhibits a linear response of oxidation peak in the entire urea concentration range of 0 $2.0 \mathrm{mM}$, and its liner relevancy can be stated as:

$$
I(\mu \mathrm{A})=16.44+68.71 C\left(\mathrm{mmol} \mathrm{L}^{-1}\right),\left(R^{2}=0.998\right)
$$

The DPV measurements manifest that the electrooxidation course keeping accordance with the switch from $\mathrm{Ni}$ (II) to Ni(III) is affected by the increasing concentration of urea and it is simultaneously electro-oxidized to nitrogen gas in this process. $^{25}$

Linear sweep voltammetry response of urea. Linear sweep voltammetry (LSV), similar to cyclic voltammetry (CV), is also a kind of common electrochemical test means, which is often used for quantitative determination of adsorbent substances. For instance, the LSV curves received on varying the concentrations of urea are shown in Fig. 4, and the red arrow head points out the orientation in which urea concentration increases constantly. The LSV map indicates that the current response strengthened progressively when various urea concentrations from 0 to $8 \mathrm{mM}$ are added. The linearly correlation can be gained from the illustration in Fig. 4, and the $R^{2}$ is as high as 0.997, proving that the Ni-MOF self-made electrode is practicable and impactful for the electrooxidation of urea.

\section{Amperometric response of urea}

The electrocatalytic capacity of urea is discriminatory with different amperometric response to $0.2 \mathrm{mM}$ urea being injected into $0.1 \mathrm{M} \mathrm{KOH}$ consecutively, under the distinct potentials $(0.50,0.55,060$ and $0.65 \mathrm{~V}$, respectively), as displayed in

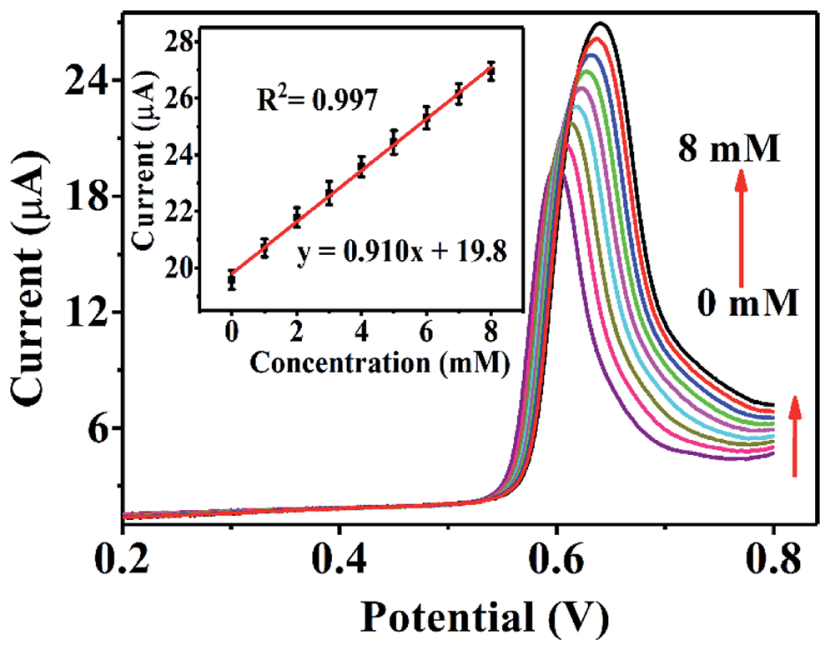

Fig. 4 LSV curves of the Ni-MOF/Nafion/GCE electrode at varying urea concentrations rang of $0-8 \mathrm{mM}$ in the potential of $0.2-0.8 \mathrm{~V}$. Inset: Mutual coherence of anode peak current responses vs. the variable concentrations.

Fig. S5A.† Fig. S5B $\uparrow$ indicates the linear relationship between the peak current and the corresponding concentration at distinct potentials. We can conclude that the potential of $+0.60 \mathrm{~V}$ was chosen as the most favorable potential because of the highest response.

The performance of the Ni-MOF/Nafion/GCE electrode as a urea sensor is evaluated by $I-T$ measurements at an oxidation peak potential of $+0.6 \mathrm{~V}$ vs. $\mathrm{Ag} / \mathrm{AgCl}$. As shown in Fig. $5 \mathrm{~A}$, the oxidation peak current heightens instantly and arrives at a plateau in about 10 seconds after the addition of urea solution each time. A calibration curve plot of the oxidation peak current response to the urea concentration is presented in the Fig. 5B. Dependency of the oxidation peak current on urea concentration $(0.01-1.0 \mathrm{mM})$ is linear with a regression equation of $I_{\mathrm{pa}}(\mu \mathrm{A})=$ $1.08+3.74 C(\mathrm{mM})$, and the $R^{2}$ is 0.992 . When the urea concentration is more than $1.0 \mathrm{mM}$, the linear analysis chart is presented in Fig. S6. $\dagger$ Dependency of the oxidation peak current on urea concentration $(1.0-7.0 \mathrm{mM})$ is linear with a regression equation of $I_{\mathrm{pa}}(\mu \mathrm{A})=4.11+0.893 C(\mathrm{mM})$, and the $R^{2}$ is 0.956 . The Ni-MOF/Nafion/GCE electrode exhibits a sensitivity of 118.77 $\mu \mathrm{A} \mathrm{mM} M^{-1} \mathrm{~cm}^{-2}$. And the lowest detection limit obtained is 2.23 $\mu \mathrm{M}$ with $\mathrm{S} / \mathrm{N}=3$, as calculated from the slope of the above linear calibration. Compared to earlier urea sensors including $\mathrm{NiO}$ and other electrodes (Table 1), this Ni-MOF nonenzymatic sensor exhibited a series of great performance, such as super sensitivity, wider linear range, and even lower detection limit.

\section{Selectivity, long-term stability and repeatability assay}

To assess the selectivity of the self-assembly sensor, we tested the current response of Ni-MOF/Nafion/GCE electrode for $0.05 \mathrm{mM}$ urea and various interfering species, such as creatinine, oxalic acid, uric acid (UA), cytosine, thymine, $\mathrm{Cl}^{-}, \mathrm{SO}_{4}{ }^{2-}$ and adenine that coexist in human urine throughout during urea estimation. The results for the mentioned study are displayed at an oxidation peak potential of $+0.6 \mathrm{~V}$ in Fig. 6. With 

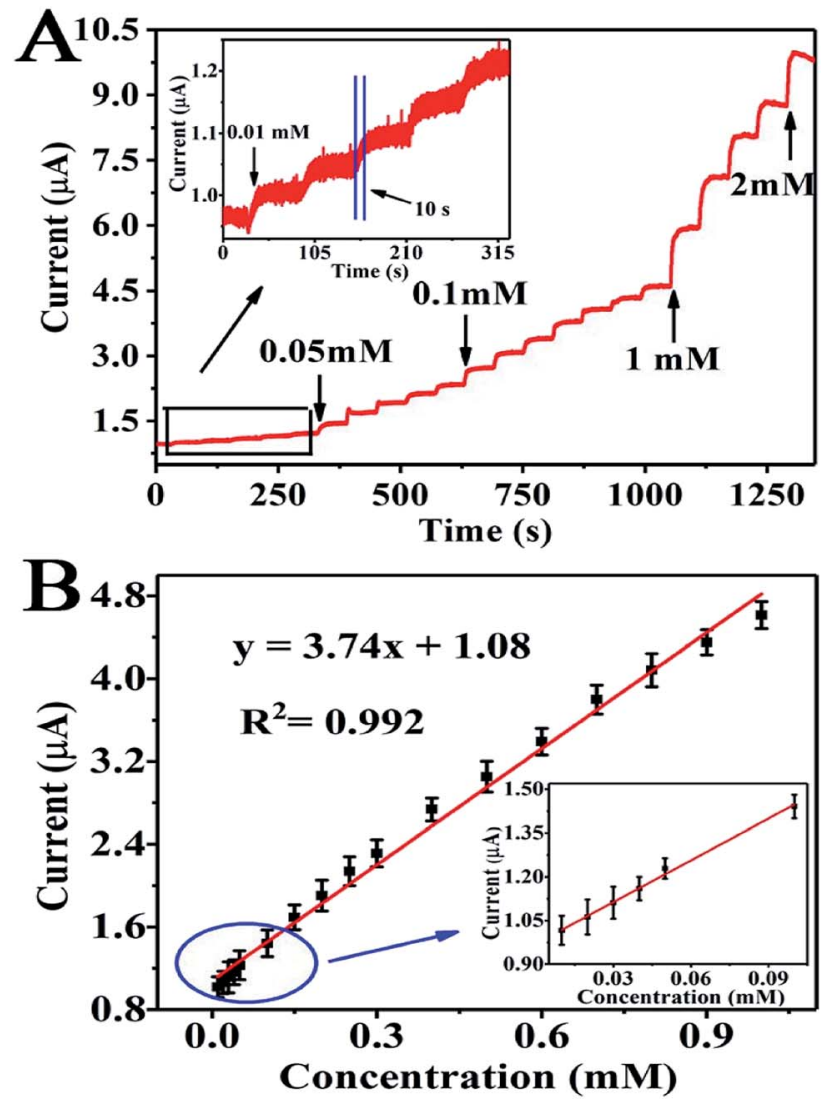

Fig. 5 (A) Amperometric $i-t$ curve for unceasing additions of urea into the electrolyte solution at oxidation peak potential of $+0.6 \mathrm{~V}$. (B) The calibration curve is shown about $I_{\mathrm{pa}}(\mu \mathrm{A})$ and $C(\mathrm{mM})$.

respective stepwise addition of creatinine, oxalic acid, UA, cytosine, thymine, $\mathrm{Cl}^{-}, \mathrm{SO}_{4}{ }^{2-}$ and adenine in $0.1 \mathrm{M} \mathrm{KOH}$ solution every 60 seconds, it is clear that the current barely changed even though the interfering species were present in 2fold or 0.2 -fold concentration levels in comparison to urea (creatinine: $10 \mu \mathrm{M}$; oxalic acid: $10 \mu \mathrm{M}$; UA: $0.1 \mathrm{mM}$; cytosine: $0.1 \mathrm{mM}$; thymine: $0.1 \mathrm{mM} ; \mathrm{Cl}^{-}: 0.1 \mathrm{mM} ; \mathrm{SO}_{4}{ }^{2-}: 0.1 \mathrm{mM}$; adenine: $0.1 \mathrm{mM}$ ). From Fig. $\mathrm{S} 7, \dagger$ we can evaluate that the current responses of all the interfering species are less than $12.5 \%$ of the urea signal. The above results are enough to prove the high selectivity of this self-assembly sensor towards urea detection.

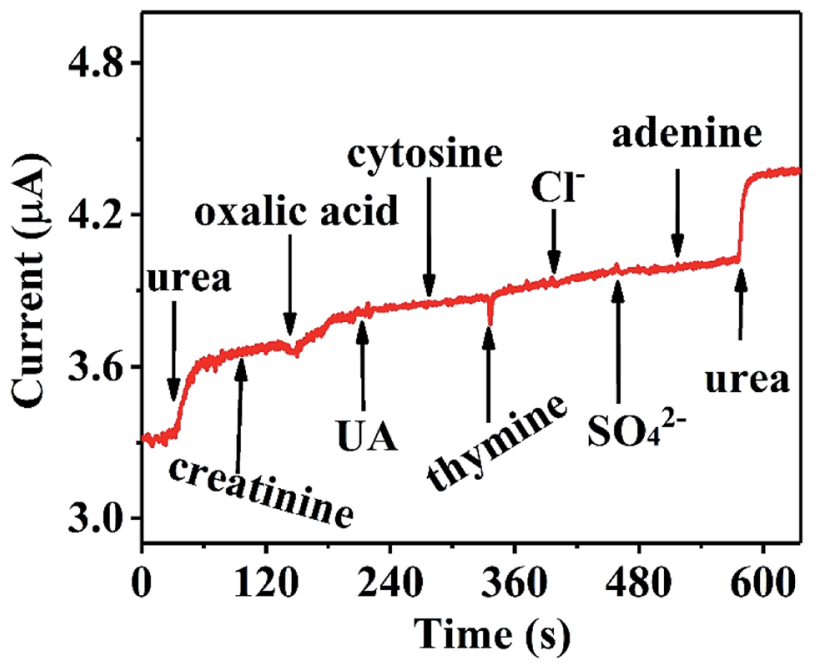

Fig. 6 The current response of the Ni-MOF/Nafion/GCE electrode to the addition of urea and different interfering species, $0.1 \mathrm{M} \mathrm{KOH}$ solution as supporting electrolyte.

Another remarkable feature of the electrodes in practical application is its prolonged stability. As displayed in Fig. $\mathrm{S} 8, \dagger$ the sensor response to urea was measured for one month. We keep the self-prepared urea sensor in the air and perform $\mathrm{CV}$ test to record the change in current value. After keeping it in the air for one week, the cathodic peak current attenuation does not reach more than $5 \%$ of the original signal. And the current response maintains $92.5 \%$ of its original current signal even after one month of air storage $\left(I / I_{0}=92.5 \%\right)$. These results demonstrate that the urea sensor based on Ni-MOF nanobelts has superior stability for sensing applications. The RSD is $2.41 \%$.

In addition, the reproducibility of the sensor is also evaluated in our study. We investigate the CV responses of five electrodes prepared with the same method in $0.1 \mathrm{M}$ electrolyte containing 1.0 $\mathrm{mM}$ urea, as displayed in Fig. S9. $\uparrow$ The five electrodes achieve illustrious consistency, where the RSD of their current response is $2.74 \%$. The excellent stability and repeatability of the Ni-MOF nanobelt electrode make them a promising candidate as a practical non-enzymatic urea sensor.

\section{Determination of urea in human body fluids}

Real urine samples were analysed by linear sweep voltammetry (LSV) to examine the practical feasibility of the Ni-MOF/Nafion/

Table 1 Comparison of analytical performances of other reported urea sensor

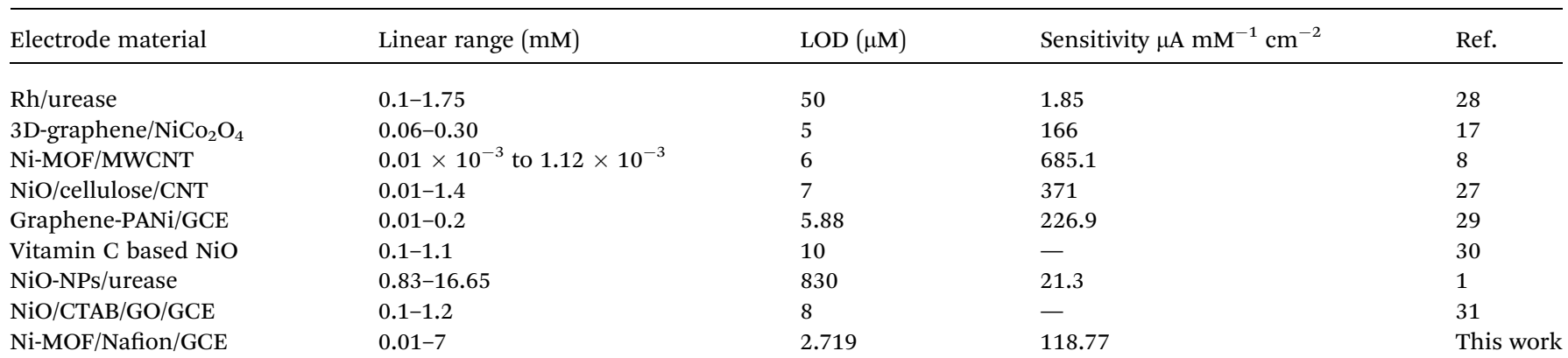




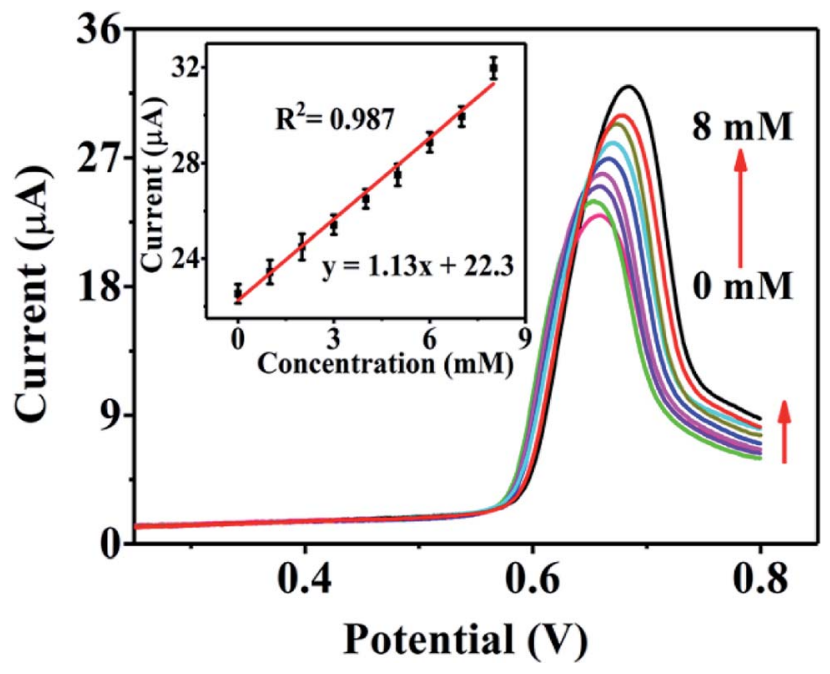

Fig. 7 LSV responses of Ni-MOF nanobelts modified GCE in diluted human urine sample containing different urea concentrations: 0, 1, 2, $3,4,5,6,7$, and $8 \mathrm{mM}$. Inset: Corresponding calibration plot of urea concentration vs. current response.

GCE electrode, as shown in Fig. 7. For large urea concentration ranging from 0 to $8 \mathrm{mM}$, the oxidation peak currents are strengthened gradually, with a correlation coefficient of the corresponding linear equation being 0.987 . In the meantime, the peak potential shifts towards the positive direction with increasing urea concentration, which indicates that other molecules in the urine have little effect on the detection of urea for this sensor. The results are satisfactory for the detection of urea in routine samples.

To further estimate the functional characteristics of Ni-MOF/ Nafion/GCE electrode in real urine samples, this developed sensor was applied to three real urine samples using the recovery test. The actual sample was diluted 100 times in $0.1 \mathrm{M}$ $\mathrm{KOH}$ solution. Then, a series of known concentration of urea solution were added to the diluted urine sample and colorimetric methods were used for a comparative study with the proposed sensor. The results are presented in Table 2 . The $t$-test results show that there is no significant difference between the two methods.
Therefore, the conclusions drawn from the Ni-MOF sensor have a certain degree of confidence. The Ni-MOF sensor shows excellent electrochemical performance towards urea. Recoveries of $92.0-115.0 \%$ verify the suitability of the developed enzymefree urea sensor in real urine samples, where the presence of various impurities has negligible effect on the authenticity of the method. In view of this, the foregoing sensor can be extended to urea determination in biological samples such as milk and serum.

\section{Conclusions}

An ultrathin Ni-MOF nanobelt decorated electrode was evaluated as a novel non-enzymatic urea sensor. The urea sensor based on Ni-MOF ultrathin nanobelts exhibits superior sensing performances such as broad detection range, low detection limit and high selectivity. Furthermore, our prepared urea sensor also shows acceptable anti-interference ability, stability and reproducibility. Overall, the urea sensor based on ultrathin Ni-MOF nanobelts is successfully fabricated and found to be promising for applications in medical diagnostics and environmental monitoring.

\section{Ethical statement}

The human urine samples were kindly provided by graduate students of our group in Shanxi Normal University (Linfen, China). In addition, the main purpose and application of this research have been informed, and the urine samples used in this experiment was obtained with the consent of the graduate students (Qiangqiang Niu and Zi-Ang Chen, respectively).

\section{Conflicts of interest}

There are no conflicts to declare.

\section{Acknowledgements}

This work was supported by the National Natural Science Foundation of China (No. 21705103), the Applied Basic Research Project of Shanxi Province (No. 201801D221392), the

Table 2 Determination and recovery results of urea in urine samples by the developed sensor

\begin{tabular}{|c|c|c|c|c|c|}
\hline Sample no. & Determined by standard method ${ }^{a}(\mathrm{mM})$ & $\begin{array}{l}\text { Determined by } \\
\text { sensor }(\mathrm{mM})\end{array}$ & $\begin{array}{l}\text { Urea spiked } \\
\text { sample }(\mathrm{mM})\end{array}$ & Founded by sensor (mM) & Recovery (\%) \\
\hline \multirow[t]{2}{*}{ Sample-1 } & \multirow[t]{2}{*}{$3.19^{a}$} & \multirow[t]{2}{*}{2.98} & 1.00 & 3.97 & 99.5 \\
\hline & & & 4.00 & 7.34 & 109.0 \\
\hline \multirow[t]{2}{*}{ Sample-2 } & \multirow[t]{2}{*}{$3.75^{a}$} & \multirow[t]{2}{*}{3.20} & 1.00 & 4.12 & 92.0 \\
\hline & & & 3.00 & 6.21 & 100.3 \\
\hline & \multirow{2}{*}{$2.43^{a}$} & \multirow{2}{*}{2.71} & 3.00 & 5.94 & 107.7 \\
\hline & & & 4.00 & 7.00 & 107.25 \\
\hline
\end{tabular}

${ }^{a}$ Determined by colorimetric method. ${ }^{32}$ 
Graduate Education Innovation Project of Shanxi Province (2018SY057), Collaborative Innovation Center for Shanxi Advanced Permanent Materials (2019-05) and Technology and the 1331 Engineering of Shanxi Province. We appreciate Prof. Dr Xiufang Qin (Shanxi Normal University) for her help on TEM and XRD characterizations. At the same time, I sincerely deliver my thanks to graduate students of our group in Shanxi Normal University (Linfen, China) for providing human urine samples.

\section{Notes and references}

1 M. Tyagi, M. Tomar and V. Gupta, NiO nanoparticle-based urea biosensor, Biosens. Bioelectron., 2013, 41, 110-115.

2 M. Singh, N. Verma, A. K. Garg and N. Redhu, Urea biosensors, Sens. Actuators, B, 2008, 134, 345-351.

3 G. Dhawan, G. Sumana and B. D. Malhotra, J. Biochem. Eng., 2009, 44, 42-52.

$4 \mathrm{M}$. Gutierrez, S. Alegret and M. Valle, Potentiometric bioelectronic tongue for the analysis of urea and alkaline ions in clinical samples, Biosens. Bioelectron., 2007, 22, 2171-2178.

5 B. Lakard, G. Herlem, S. Lakard, A. Antoniou and B. Fahys, Urea potentiometric biosensor based on modified electrodes with urease immobilized on polyethylenimine films, Biosens. Bioelectron., 2004, 19, 1641-1647.

6 A. Azadbakht and M. B. Gholivand, Covalent attachment of Ni-2,3-pyrazine dicarboxylic acid onto gold nanoparticle gold electrode modified with penicillamine CdS quantum dots for electrocatalytic oxidation and determination of urea, Electrochim. Acta, 2014, 125, 9-21.

7 A. Mahmood, W. Guo, H. Tabassum and R. Zou, MetalOrganic Framework-Based Nanomaterials for Electrocatalysis, Adv. Energy Mater., 2016, 1600423.

8 Y. Miao, L. Ouyang, S. Zhou, L. Xu, Z. Yang, M. Xiao and R. Ouyang, Electrocatalysis and electroanalysis of nickel, its oxides, hydroxides and oxyhydroxides toward small molecules, Biosens. Bioelectron., 2014, 53, 428-439.

9 N. S. Nguyen and H. H. Yoon, Nickel oxide-deposited cellulose/CNT composite electrode for non-enzymatic urea detection, Sens. Actuators, B, 2016, 236, 304-310.

10 R. H. Tammam and M. M. Saleh, On the electrocatalytic urea oxidation on nickel oxide nanoparticles modified glassy carbon electrode, J. Electroanal. Chem., 2017, 794, 189-196.

11 Q. Wang, Y. Xue, S. Sun, S. Yan, H. Miao and Z. Liu, Facile synthesis of ternary spinel Co-Mn-Ni nanorods as efficient bi-functional oxygen catalysts for rechargeable zinc-air batteries, J. Power Sources, 2019, 435, 226761.

12 Y.-H. Chung, K. Gupta, J.-H. Jang, H. S. Park, I. Jang, J. H. Jang, Y.-K. Lee, S.-C. Lee and S. J. Yoo, Rationalization of electrocatalysis of nickel phosphide nanowires for efficient hydrogen production, Nano Energy, 2016, 26, 496503.

13 P.-Q. Liao, J.-Q. Shenand and J.-P. Zhang, Metal-organic frameworks for electrocatalysis, Coord. Chem. Rev., 2018, $373,22-48$.
14 H. Liu, W. Ding, S. Lei, X. Tian and F. Zhou, Selective adsorption of $\mathrm{CH}_{4} / \mathrm{N}_{2}$ on Ni-based MOF/SBA-15 composite materials, Nanomaterials, 2019, 9, 149.

15 D. Zhu, C. Guo, J. Liu, L. Wang, Y. Duand and S.-Z. Qiao, Two-dimensional metal-organic frameworks with high oxidation states for efficient electrocatalytic urea oxidation, Chem. Commun., 2017, 53, 10906-10909.

16 J.-Y. Zhang, T. He, M. Wang, R. Qi, Y. Yan, Z. Dong, H. Liu, H. Wang and B. Y. Xia, Energy-saving hydrogen production coupling urea oxidation over a bifunctional nickelmolybdenum nanotube array, Nano Energy, 2019, 60, 894902.

17 T. Q. N. Tran, G. Das and H. H. Yoon, Nickel-metal organic framework/MWCNT composite electrode for nonenzymatic urea detection, Sens. Actuators, B, 2017, 243, 7883.

18 X. Xiao, S. Zheng, X. Li, G. Zhang, X. Guo, H. Xue and H. Pang, Facile synthesis of ultrathin Ni-MOF nanobelts for high-efficiency determination of glucose in human serum, J. Mater. Chem. B, 2017, 5, 5234-5239.

19 H. Yan, J. Bai, J. Wang, X. Zhang, B. Wang, Q. Liu and L. Liu, Graphene homogeneously anchored with $\mathrm{Ni}(\mathrm{OH})_{2}$ nanoparticles as advanced supercapacitor electrodes, CrystEngComm, 2013, 15, 10007-10015.

20 J. Yang, P. Xiong, C. Zheng, H. Qiu and M. Wei, Metalorganic frameworks: a new promising class of materials for a high-performance supercapacitor electrode, J. Mater. Chem. A, 2014, 2, 16640-16644.

21 J. Xu, C. Yang, Y. Xue, C. Wang, J. Cao and Z. Chen, Facile synthesis of novel metal-organic nickel hydroxide nanorods for high performance supercapacitor, Electrochim. Acta, 2016, 211, 595-602.

22 N. I. Marzuki, F. A. Bakar, A. B. Salleh, L. Y. Heng, N. A. Yusof and S. Siddiquee, Development of Electrochemical Biosensor for Formaldehyde Determination Based on Immobilized Enzyme, Int. J. Electrochem. Sci., 2012, 7, 6070-6083.

23 S. Zhao, K. Zhang, Y. Bai, W. Yang and C. Sun, Glucose oxidase/colloidal gold nanoparticles immobilized in Nafion film on glassy carbon electrode: direct electron transfer and electrocatalysis, Bioelectrochemistry, 2006, 69, 158-163.

$24 \mathrm{~V}$. Vedharathinam and G. G. Botte, Understanding the electro-catalytic oxidation mechanism of urea on nickel electrodes in alkaline medium, Electrochim. Acta, 2012, 81, 292-300.

25 A. G. Meguerdichian, T. Jafari, M. R. Shakil, R. Miao, L. A. Achola, J. Macharia, A. A. Shirazi and S. L. Suib, Synthesis and Electrocatalytic Activity of Ammonium Nickel Phosphate, $\left[\mathrm{NH}_{4}\right] \mathrm{NiPO}_{4} \cdot 6 \mathrm{H}_{2} \mathrm{O}$, and $\beta$ Nickel Pyrophosphate, $\beta \mathrm{Ni}_{2} \mathrm{P}_{2} \mathrm{O}_{7}$ : Catalysts for Electrocatalytic Decomposition of Urea, Inorg. Chem., 2018, 57, 1815-1823.

26 B. K. Boggs, R. L. King and G. G. Botte, Urea electrolysis: direct hydrogen production from urine, Chem. Commun., 2009, 32, 4859-4861.

27 Y. Velichkova, Y. Ivanov, I. Marinov, R. Ramesh, N. R. Kamini, N. Dimcheva, E. Horozova and T. Godjevargova, Amperometric electrode for 
determination of urea using electrodeposited rhodium and immobilized urease, J. Mol. Catal. B: Enzym., 2011, 69, 168175.

28 N. S. Nguyen, G. Das and H. H. Yoon, Nickel/cobalt oxidedecorated 3D graphene nanocomposite electrode for enhanced electrochemical detection of urea, Biosens. Bioelectron., 2016, 77, 372-377.

29 R. Sha, K. Komori and S. Badhulika, Graphene-polyaniline composite based ultra-sensitive electrochemical sensor for non-enzymatic detection of urea, Electrochim. Acta, 2017, 233, 44-51.

30 M. Arain, A. Nafady, S. Sirajuddin, Z. H. Ibupoto, S. T. H. Sherazi, T. Shaikh, H. Khan, A. Alsalme, A. Niazf and M. Willander, Simpler and highly sensitive enzymefree sensing of urea via $\mathrm{NiO}$ nanostructures modified electrode, $R S C A d v .$, 2016, 6, 39001-39006.

31 Z. Parsaee, Synthesis of novel amperometric urea-sensor using hybrid synthesized NiONPs/GO modified GCE in aqueous solution of cetrimonium bromide, Ultrason. Sonochem., 2018, 44, 120-128.

32 M. Baumgratner, M. Flock, P. Winter, W. Luf and W. Baumgartner, Evaluation of flow injection analysis for determination of urea in sheep's and cow's milk, Acta Vet. Hung., 2002, 50, 263-271. 